\title{
Hour time-scale QPOs in the X-ray and radio emission of LS I +61 ${ }^{\circ} 303$
}

\author{
S. Nösel, ${ }^{1}$ R. Sharma, ${ }^{1 \star}$ M. Massi,${ }^{1}$ G. Cimò ${ }^{2,3}$ and M. Chernyakova ${ }^{4,5}$ \\ ${ }^{1}$ Max-Planck-Institut für Radioastronomie, Auf dem Hügel 69, D-53121 Bonn, Germany \\ ${ }^{2}$ Joint Institute for VLBI ERIC, P.O. Box 2, 7990 AA Dwingeloo, The Netherlands \\ ${ }^{3}$ Netherlands Institute for Radio Astronomy, P.O. Box 2, 7990 AA Dwingeloo, The Netherlands \\ ${ }^{4}$ School of Physical Sciences and C-fAR, Dublin City University, Dublin 9, Ireland \\ ${ }^{5}$ Dublin Institute for Advanced Studies, 31 Fitzwilliam Place, Dublin 2, Ireland
}

Accepted XXX. Received YYY; in original form ZZZ

\begin{abstract}
LS I $+61^{\circ} 303$ is an X-ray binary with a radio outburst every $\sim 27$ days. Previous studies of the stellar system revealed radio microflares superimposed on the large radio outburst. We present here new radio observations of LS I $+61^{\circ} 303$ at $2.2 \mathrm{GHz}$ with the Westerbork Synthesis Radio Telescope (WSRT). Using various timing analysis methods we find significant Quasi-Periodic Oscillations (QPOs) of 55 minutes stable over the duration of 4 days. We also use archival data obtained from the Suzaku satellite at X-ray wavelengths. We report here for the first time significant X-ray QPOs of about 2 hours present over the time span of 21 hours. We compare our results with the previously reported QPO observations and we conclude that the QPOs seem to be associated with the radio outburst, independent of the amplitude of the outburst. Finally, the different QPO time-scales are discussed in the context of magnetic reconnection.
\end{abstract}

Key words: Radio continuum: stars - X-rays: binaries - X-rays: individual (LS I +61³03)

\section{INTRODUCTION}

Microquasars are excellent laboratories to probe the accretionejection coupling in accreting compact objects. Microquasars are radio emitting X-ray binaries, i.e., stellar systems where a compact object (a neutron star or a black hole) accretes matter from a normal star. From the accretion disc and inflow, which emits in X-rays, a relativistic jet is launched which emits in the radio band (Mirabel \& Rodríguez 1999).

Timing analysis of variability is a powerful tool to investigate variations around the accretion flow and their relationship with variations in the jet. In fact, in one microquasar, the black hole Xray binary GRS 1915+105, a link between Quasi-Periodic Oscillations (QPOs) in the accretion flow and in the jet has been observed. Radio oscillations on time-scales of 20-40 minutes have been clearly related to quasi-periodic dips in the X-ray light curve (see Fig. 5 in Pooley \& Fender 1997, Fig. 3 in Mirabel et al. 1998, Fig. 3 in Klein-Wolt et al. 2002). Belloni et al. (1997) suggested that the X-ray dips correspond to the disappearance of the inner part of the accretion disc. The relation of the radio oscillations to these dips led to the hypothesis that at least some of the material from the inner disc had in fact been ejected from the system, instead of simply

^ E-mail: rsharma@mpifr-bonn.mpg.de falling into the black hole (see references in Mirabel \& Rodríguez 1999).

Variability with time-scales of minutes-hours has been observed only in few microquasars: V404 Cyg (Han \& Hjellming 1992), Cygnus X-1 (Marti et al. 2001), GRS 1915+105 (e.g. Rodríguez \& Mirabel 1997; Fender et al. 1997, 2002) and LS I +61 303 (e.g. Peracaula et al. 1997; Taylor et al. 1992). Among them, the binary stellar system LS I $+61^{\circ} 303$ with its strong and periodic radio outbursts (Gregory 2002; Massi \& TorricelliCiamponi 2016) represents a favourable target for QPO observations. Previously, one detection of radio QPO (Peracaula et al. 1997) and hints for X-ray QPO (Harrison et al. 2000) with timescales of minutes-hours were observed in LS I $+61^{\circ} 303$.

The aim of this work is to verify the suggested presence of QPOs with time-scales of minutes-hours in the X-ray emission of LS I $+61^{\circ} 303$ and corroborate the presence of radio QPOs with the same time-scales.

\subsection{Previously reported variability in $L S I+61^{\circ} 303$}

The stellar binary system LS I $+61^{\circ} 303$ is a high-mass X-ray binary (HMXB) comprising of a massive star that shows optical spectra of a B0 V star (Casares et al. 2005) and a compact object. As shown in Massi et al. (2017), LS I $+61^{\circ} 303$ fits the trend of intermediate luminosity accreting black holes, the systems where the X-ray 

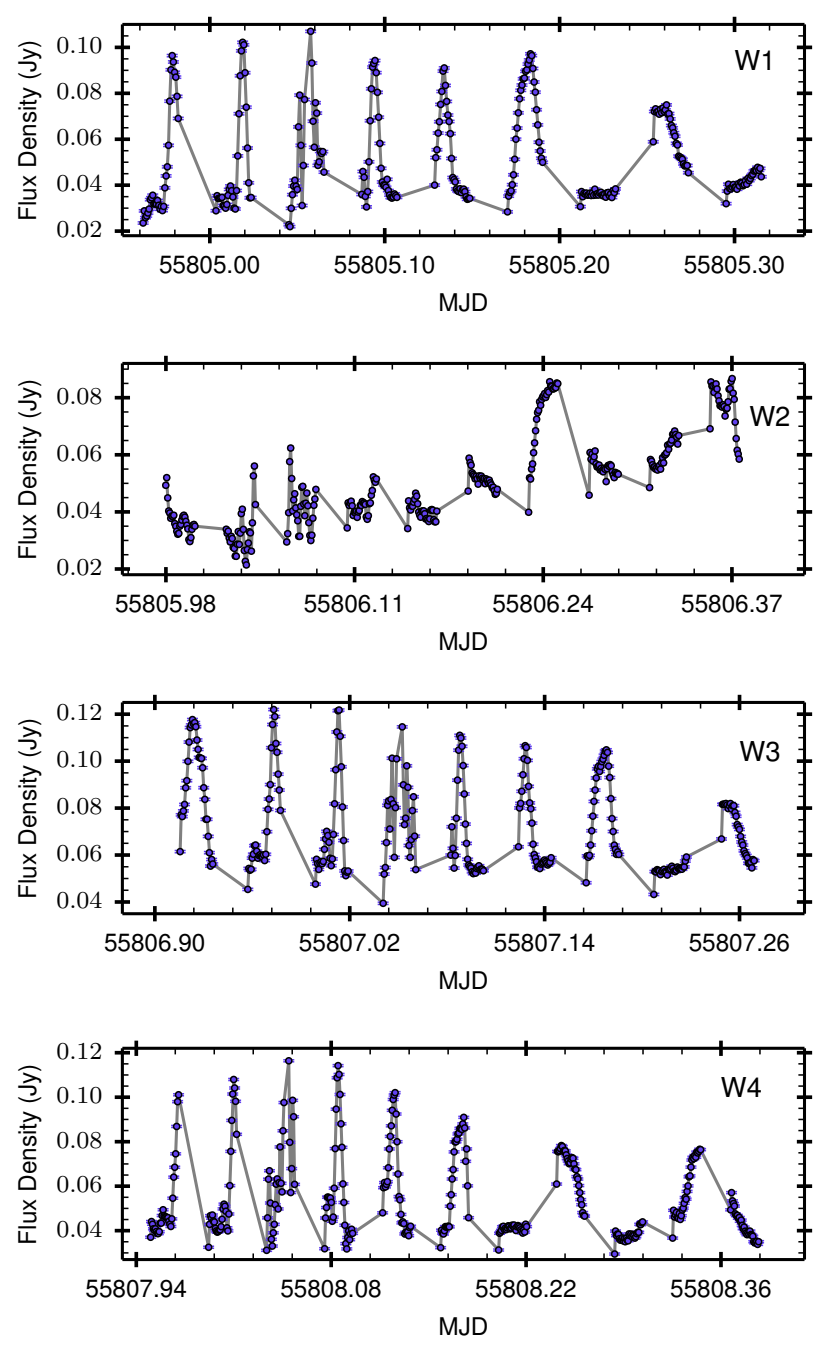

Figure 1. Top to bottom: Radio light curves of LS I $+61^{\circ} 303$ at $2.2 \mathrm{GHz}$ for all four days of observations with WSRT. Distinct microflares are already visible at all days with 7,8 and 8 microflares for observations W1, W3 and W4, respectively. Observation W2 show hints of small microflares. The grey line is used to connect the data points.

spectrum is thought to originate from inverse Compton processes in their inner accretion flow (Yang et al. 2015a,b). LS I +61 303 is located at a distance of $2.0 \pm 0.2 \mathrm{kpc}$ (Frail \& Hjellming 1991) and has an orbital period, $P_{\text {orb }}=26.4960 \pm 0.0028$ days (Gregory 2002). Its orbital phase is defined as $\Phi_{\text {orb }}=\frac{t-t_{0}}{P_{\text {orb }}}-\operatorname{int}\left(\frac{t-t_{0}}{P_{\text {orb }}}\right)$, where, $t_{0}=$ MJD 43366.275. The source has a radio outburst every $26.70 \pm 0.05$ days (Ray et al. 1997; Jaron \& Massi 2013). The radio outburst in LS I $+61^{\circ} 303$ is also modulated over a long-term period, $P_{\text {long }}=1626 \pm 68$ days (Massi \& Torricelli-Ciamponi 2016). The superorbital phase is defined analogous to the orbital phase as, $\Theta=\frac{t-t_{0}}{P_{\text {long }}}-\operatorname{int}\left(\frac{t-t_{0}}{P_{\text {long }}}\right)$.

Previous studies of this source have revealed short-term radio variability superimposed on the radio outburst. For the first time, a step-like pattern was observed in LS I $+61^{\circ} 303$ with the Westerbork Synthesis Radio Telescope (WSRT) phased array with variability time-scale of $\sim 10^{3}$ seconds during the decay of the radio outburst
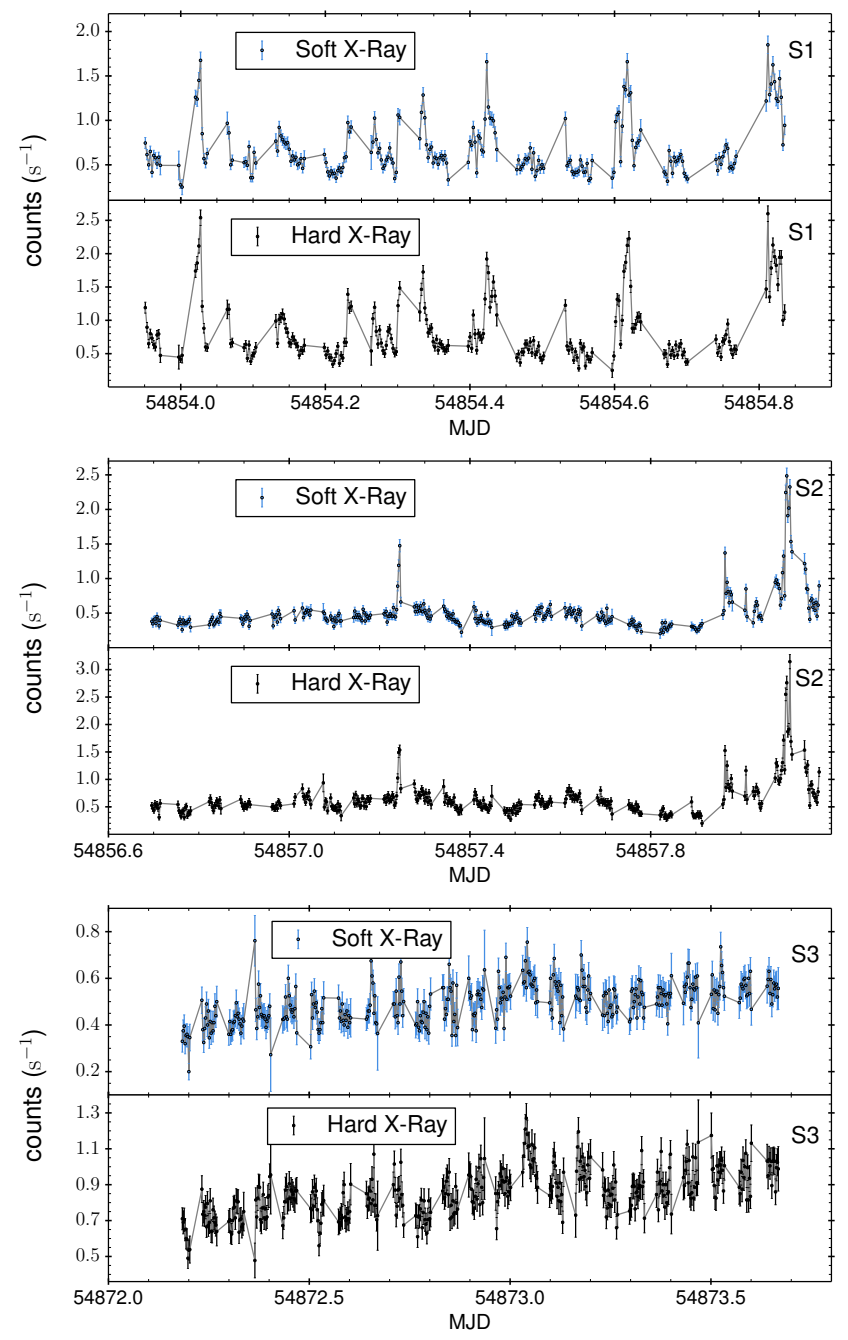

Figure 2. Top to bottom: X-ray light curves of LS I +61³03 for observations done with the Suzaku satellite. Soft X-ray data are represented in blue colour and hard X-ray data in black colour. The grey line is used to connect the data points. Timing analysis reveals QPOs only for observation S1.

(Taylor et al. 1992). The first systematic analysis of short-term variability in LS I $+61^{\circ} 303$ was performed using VLA observations by Peracaula et al. (1997), wherein microflares with recurrence period of 1.4 hours were reported to be active for $\sim 8$ hours with an amplitude of $\sim 4 \mathrm{mJy}$ during the decay of the observed outburst. At higher energy, Harrison et al. (2000) indicated 30-40 minutes variability in one of the $\mathrm{X}$-ray observations done with the Advanced Satellite for Cosmology and Astrophysics (ASCA) and associated with the onset of a radio outburst. Longer time-scale radio QPOs of 15.4 hours and 2 days were observed by Jaron et al. (2017) and Zimmermann et al. (2015), respectively.

In Sect. 2, we describe new observations and their data analysis. The results of the analysis are presented in Sect. 3. In Sect. 4, we compare our results with the previous QPO observations of the source. Finally, we conclude and discuss our results in Sect. 5. 
Table 1. Log of WSRT and Suzaku data used in this work.

\begin{tabular}{lcccccc}
\hline Telescope & Obs. starting date & Label & MJD & $\Phi_{\text {orb }}$ & $\Theta$ & $P_{\mathrm{QPO}}$ \\
\hline WSRT $(2.2 \mathrm{GHz})$ & $31.08 .2011-03.09 .2011$ & W1-W4* & $55804.941-55807.929$ & $0.45-0.57$ & $0.650-0.652$ & $55.3 \pm 0.3 \mathrm{~min}$ \\
Suzaku & 22.01 .2009 & $\mathrm{~S} 1$ & 54853.951 & 0.56 & 0.065 & $2.4 \pm 0.3 \mathrm{~h}$ \\
$(0.3-12 \mathrm{keV}$ and & 25.01 .2009 & $\mathrm{~S} 2$ & 54856.696 & 0.67 & 0.067 & No QPOs \\
$13-600 \mathrm{keV})$ & 10.02 .2009 & $\mathrm{~S} 3$ & 54872.184 & 0.25 & 0.076 & No QPOs \\
\hline
\end{tabular}

*Note: The MJD values for W1-W4 are 55804.941, 55805.959, 55806.895 and 55807.929.

Table 2. An overview of the previously reported QPOs in LS I $+61^{\circ} 303$. The references are: ${ }^{a}$ Jaron et al. (2017), ${ }^{b}$ Peracaula et al. (1997), ${ }^{\mathrm{c}}$ Taylor et al. (1992) and ${ }^{\mathrm{d}}$ Harrison et al. (2000). The occurrence of the QPOs with respect to the radio outburst evolution is given in the last column.

\begin{tabular}{lccccccc}
\hline Telescope & Obs. starting date & Label & MJD & $\Phi_{\text {orb }}$ & $\Theta$ & $P_{\mathrm{QPO}}$ & Comments \\
\hline Effelsberg a & 17.04 .2014 & $\mathrm{~J} 1$ & 56764.726 & 0.68 & 0.240 & $15.4 \pm 0.6 \mathrm{~h}$ & Decay of radio outburst. \\
$(4.85,8.35,10.45 \mathrm{GHz})$ & & & & & & & \\
VLA $^{\mathrm{b}}(5 \mathrm{GHz})$ & 06.06 .1990 & $\mathrm{P} 1$ & 48048.292 & 0.72 & 0.880 & $1.4 \mathrm{~h} ?$ & Less significant QPOs during decay. \\
& 09.09 .1993 & $\mathrm{P} 2$ & 49239.042 & 0.66 & 0.612 & $1.4 \mathrm{~h}$ & $\begin{array}{c}\text { Decay of radio outburst. } \\
\text { High flux quiescence. }\end{array}$ \\
& 13.09 .1993 & $\mathrm{P} 3$ & 49243.042 & 0.81 & 0.614 & No QPOs & $\begin{array}{c}\text { Decay of radio outburst. } \\
\text { EVN }^{\mathrm{c}}(5 \mathrm{GHz})\end{array}$ \\
$\mathrm{ASCA}^{\mathrm{d}}(1-5 \mathrm{keV})$ & 01.10 .1987 & $\mathrm{~T} 1$ & 47069.875 & 0.78 & 0.278 & $1000 \mathrm{~s}$ & Before onset of radio outburst. \\
& 03.02 .1994 & $\mathrm{H} 1$ & 49386.104 & 0.20 & 0.702 & No QPOs & Onset of radio outburst. \\
\hline
\end{tabular}

\section{OBSERVATIONS AND DATA ANALYSIS}

\subsection{New WSRT radio observations}

The here presented WSRT observations of LS I $+61^{\circ} 303$ at $2.2 \mathrm{GHz}$ are part of a larger observation whose data reduction is still in progress. The source was observed for four consecutive days (see Table 1) from August 31, 2011 until September 3, 2011. We obtained 9-11 scans each of 30 minute length. The integration time was 1 minute. The data reduction of the raw data from the telescope was performed using the Common Astronomy Software Package $^{1}$ (CASA). We used $3 \mathrm{C} 48$ as a flux calibrator. The obtained light curves are shown in Fig. 1 for all four days.

\subsection{X-ray observations with Suzaku}

For X-ray observations of LS I $+61^{\circ} 303$ we use the archival data obtained from the Suzaku satellite (Chernyakova et al. 2017). The data consist of three observation runs in the year 2009 (see Table 1), where each observation run includes data of soft X-ray (0.3$12 \mathrm{keV}$ ) and hard X-ray (13-600 keV). The total duration of observation for each run varies, with 21.3 hours for S1, 35.5 hours for $\mathrm{S} 2$ and 35.6 hours for S3. The data reduction was done using the $\mathrm{HEASOFT}^{2}$ v.6.16 software package and the spectral modelling was performed in the XSPEC environment v.12.8.2. For more details on the data reduction see Sect. 2.1 in Chernyakova et al. (2017). The data were integrated over 200 seconds. The obtained light curves are shown in Fig. 2.

\subsection{Timing analysis}

To find variability on short time-scales in LS I $+61^{\circ} 303$ at both radio and X-ray wavelengths, we use three timing analysis techniques. The data were initially analysed using the Lomb-Scargle

1 For detailed information about CASA visit https://casa.nrao.edu.

2 http://heasarc.gsfc.nasa.gov/docs/software/lheasoft/. periodogram which is a powerful tool to find and test the significance of weak periodic signals in unevenly sampled data (Lomb 1976; Scargle 1982). The significance of the found periodic signal is tested with the Fischer-randomization test where the flux is permuted thousand times and thousand new randomised time series are created and their periodograms calculated (Linnell Nemec \& Nemec 1985). The proportion of permuted time series that contain a higher peak in the periodogram than the original periodogram at any frequency then gives the false alarm probability, $p$ of the peak. If $p<0.01$, the period is significant and if $0.01<p<0.1$ the period is marginally significant. We then analysed the data using the phase dispersion minimization (PDM) method which is useful when we have a limited number of data points available and the light curve is non-sinusoidal (Stellingwerf 1978). In this technique, the data-series is folded on many trial frequencies. The folded data are then divided into various bins and their variance is calculated. The PDM statistics is the ratio of overall variance of different bins and the variance of the original data. If the trial period is a true period, then we observe a local minimum in the PDM which approaches zero. The minima in the PDM must be an approximate mirror image of the maxima in the Lomb-Scargle periodogram. Finally, the data were folded in phase averaged bins with the significant period, $P$, found from both the above explained methods. The phase $\phi$ is calculated as $\phi=\frac{t-t_{0}}{P}-\operatorname{int}\left(\frac{t-t_{0}}{P}\right)$, where, $t_{0}$ is the first data point of each observation.

\section{RESULTS}

\subsection{Variability in radio}

The light curves for the $2.2 \mathrm{GHz}$ data (see Fig. 1) show well sampled microflares with varying amplitude and width. Observations $\mathrm{W} 1, \mathrm{~W} 3$ and $\mathrm{W} 4$ show 7, 8 and 8 distinct microflares, respectively. Observation W2 shows only hints of small microflares. The LombScargle periodogram of the combined dataset of four days is shown in the top panel of Fig. 3. We find a significant periodic signal in the data with a period of $55.3 \pm 0.2$ minutes. The PDM statistics (see 

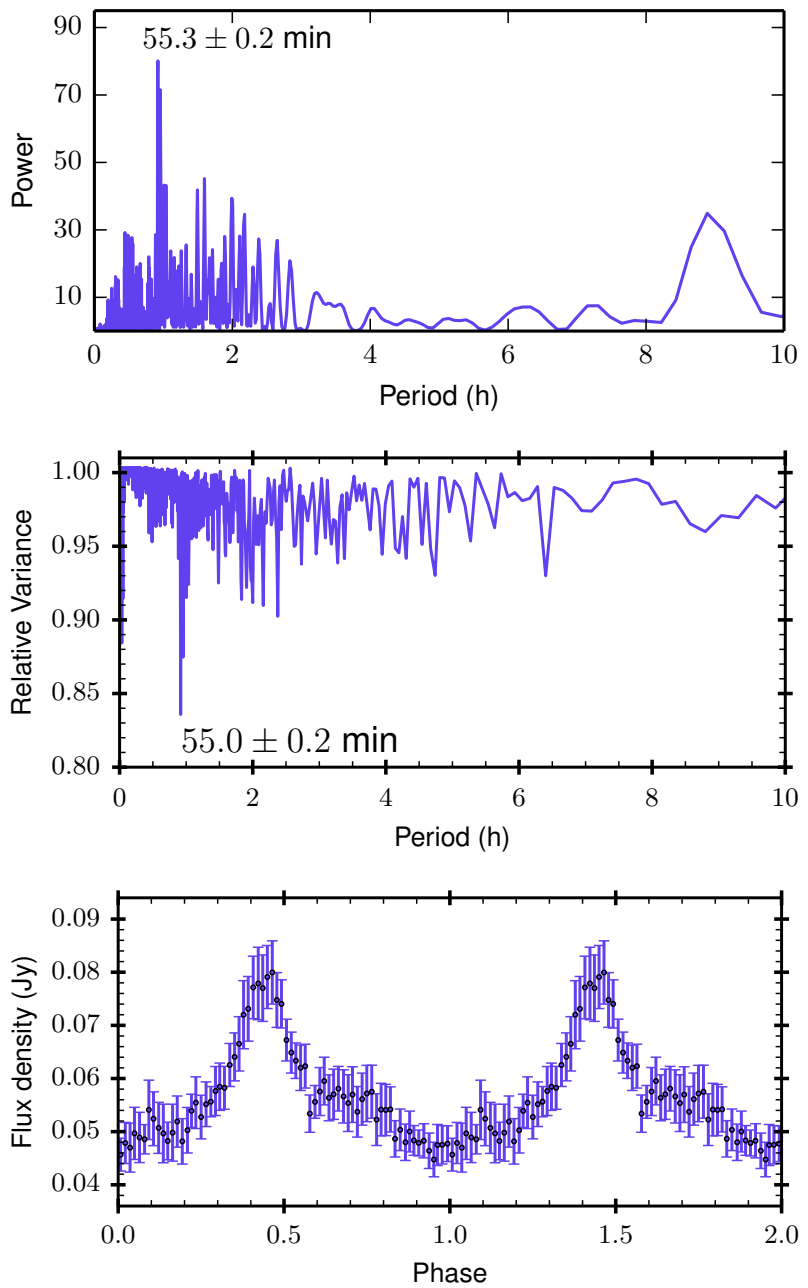

Figure 3. Timing analysis results of the radio $(2.2 \mathrm{GHz})$ data observed with the WSRT for all four days. Top: Lomb-Scargle periodogram. Middle: The true period yields the minimum relative variance in the PDM curve. Bottom: Phase averaged data folded with the significant period of 55.3 minutes and with a phase bin size of 0.014 . For clarity, the data are repeated in the second cycle.

middle panel of Fig. 3) gives a significant period of $55.0 \pm 0.2 \mathrm{~min}-$ utes. Finally, the phase averaged data folded with the found period from the periodogram are shown in the bottom panel of Fig. 3. The well clustered data peaking at one phase confirms the presence of the period of 55.3 minutes over four days.

\subsection{Variability in X-rays}

The light curves for both soft and hard X-ray (see Fig. 2) show at least 7 distinct flares for S1. The observation S2 was done three days later and shows one isolated flare followed by the other two flares. The observation S3 which was done 19 days later has hardly any visible flares. The timing analysis results of soft and hard Xray data are similar and therefore we show the results of soft Xrays only. For S1, the Lomb-Scargle periodogram is shown in the top panel of Fig. 4 with a significant period of $2.4 \pm 0.1$ hours. The PDM statistics (see the middle panel of Fig. 4) reveal a significant period of $2.3 \pm 0.1$ hours. The red line in the top and in the mid-
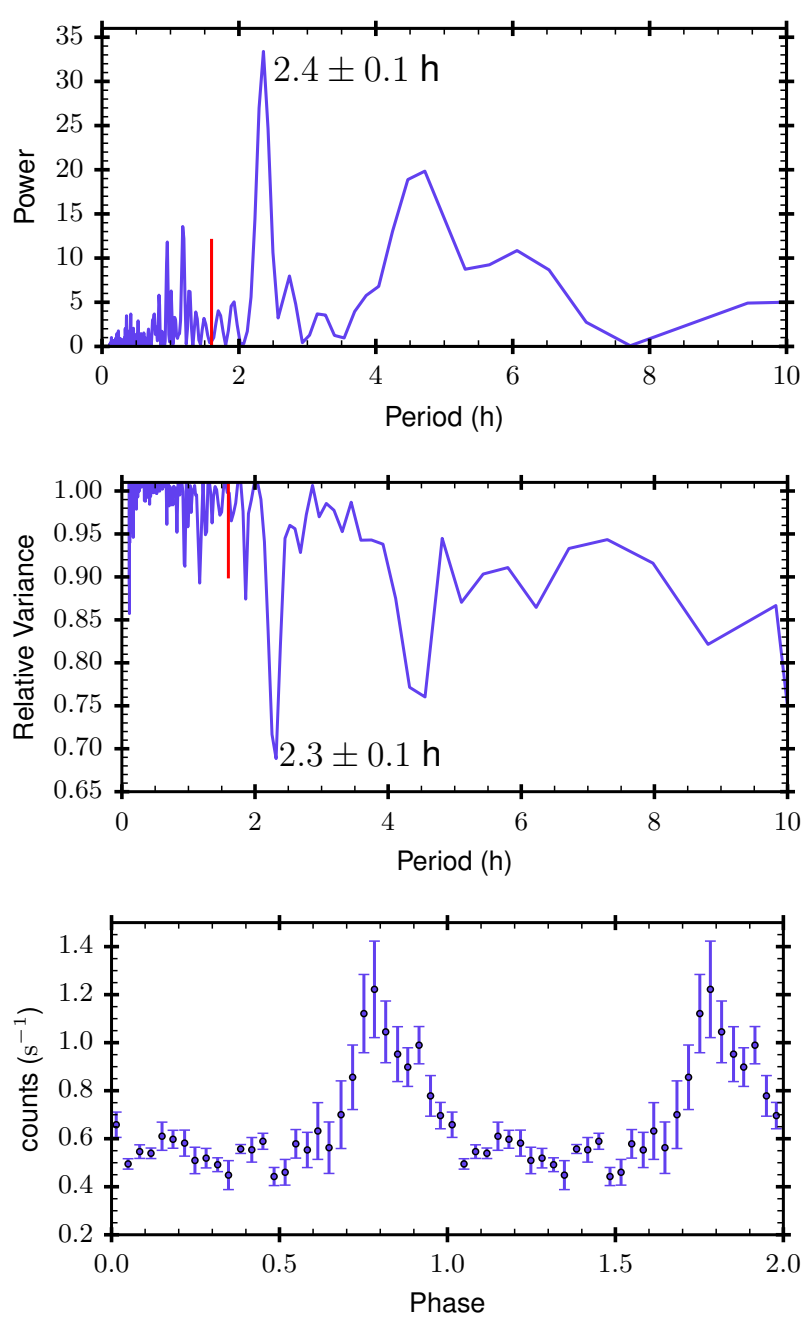

Figure 4. Timing analysis results of the soft X-ray $(0.3-12 \mathrm{keV})$ data observed with the Suzaku satellite for the first run of the observation session (S1). Top: Lomb-Scargle periodogram. The red line indicates the orbital period of 1.6 hours of the satellite. Middle: The true period yields the minimum relative variance in PDM and is specular of the peak in LombScargle periodogram. Bottom: Phase averaged data folded with the significant period of 2.4 hours and with a phase bin size of 0.033 . For clarity, the data are repeated in the second cycle.

dle panel of Fig. 4 indicates the orbital period of 1.6 hours of the Suzaku satellite, which does not interfere with our results. Finally, the phase averaged data folded with the found period are shown in the bottom panel of Fig. 4. The data are well clustered at one phase which confirms the period of 2.4 hours. For S2 and S3, no QPOs were found during the analysis.

\section{COMPARISON OF OUR RESULTS WITH PREVIOUS QPO OBSERVATIONS}

In this work we report about two new observations of LS I $+61^{\circ} 303$ with QPOs, one at radio and one at X-ray wavelengths. In order to understand the implications of our results, we compare them with the previously reported QPOs. Table 2 gives an overview of the observations done in the past with a comparison between their orbital 

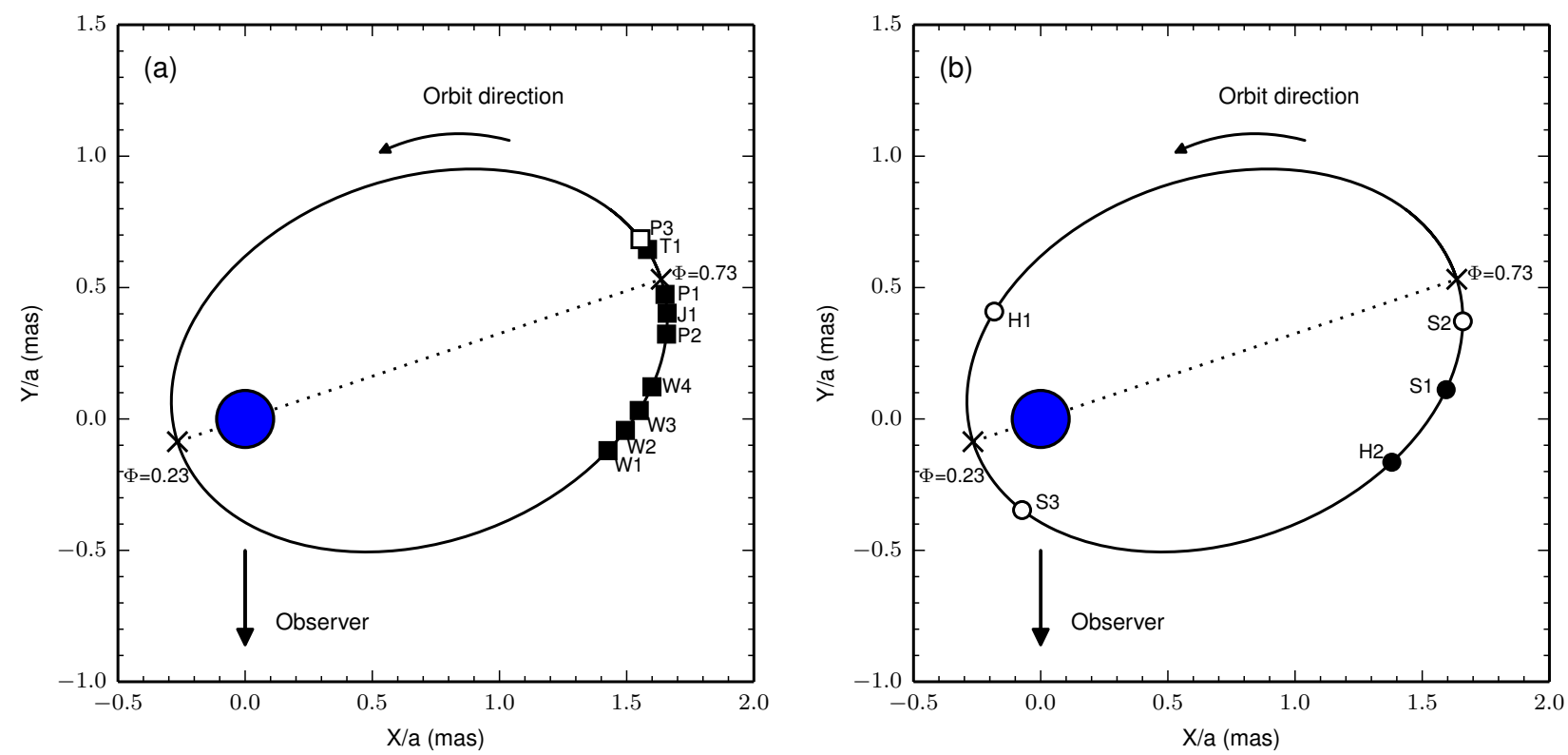

Figure 5. (a) Radio observations (squares) and (b) X-ray observations (circles) as given in Table 1 and Table 2 marked here along the relative orbit of the compact object in LS $\mathrm{I}+61^{\circ} 303$. The crosses mark the periastron and apoastron phase intervals. The QPO detections are represented by black squares and circles, and non-detections are represented by open squares and circles. The orbital parameters are taken from Casares et al. (2005) with the Be star at ellipse focus $(0,0)$
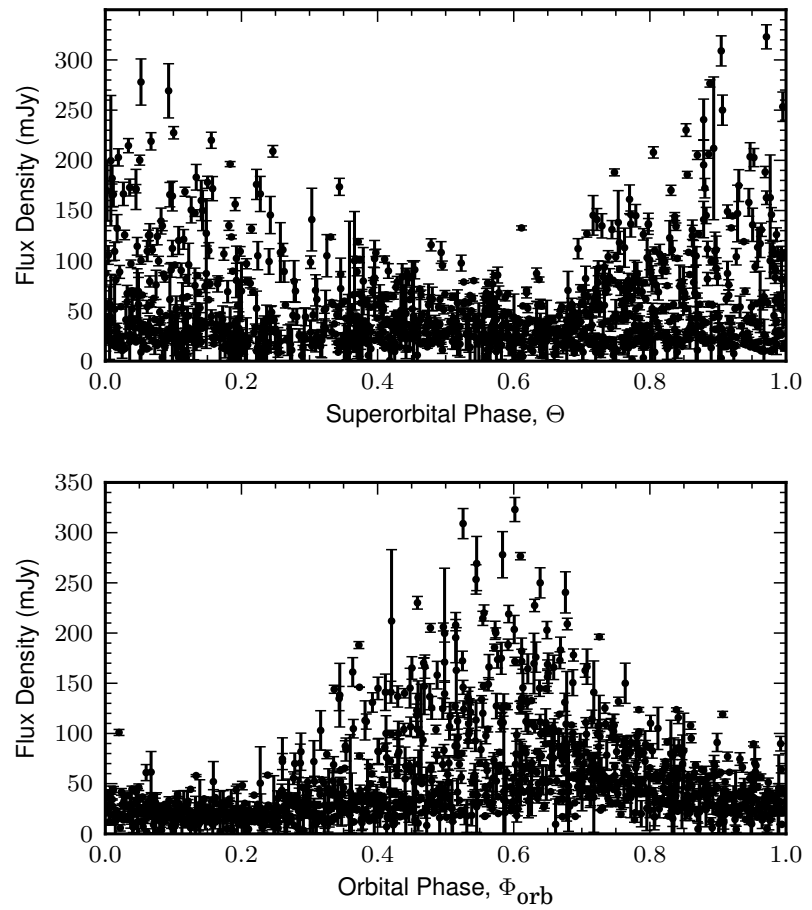

Figure 6. Radio data of LS I $+61^{\circ} 303$ of $\sim 37$ years from Massi \& TorricelliCiamponi (2016). Top: Radio data folded with the superorbital period of 1626 days. The radio flux modulates with minimum flux around $\Theta \sim 0.6$ and maximum flux around $\Theta \sim 0.1$. Bottom: Radio data folded with the orbital period of 26.496 days with flux density peak observed at $\Phi_{\text {orb }} \sim 0.6$.

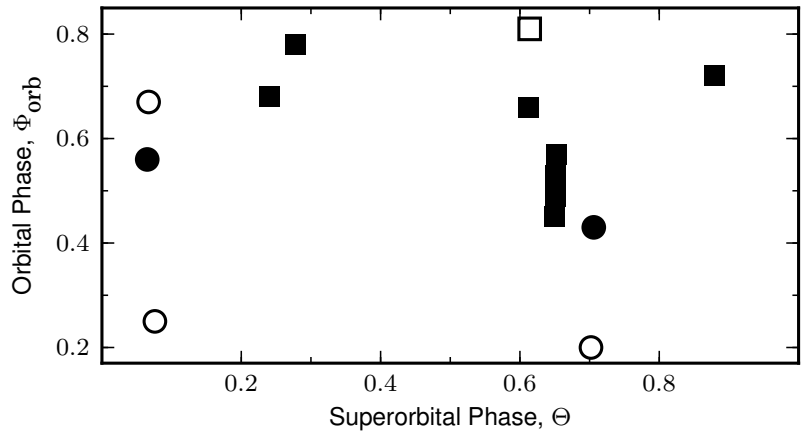

Figure 7. Radio and X-ray observations as in Table 1 and Table 2 represented with their corresponding orbital phase and superorbital phase. The radio and X-ray observations are represented as squares and circles, respectively. The colour coding is same as in Fig. 5.

phase, superorbital phase and period of QPO $\left(P_{\mathrm{QPO}}\right)$. In Fig. 5, we present the relative orbital geometry of LS I $+61^{\circ} 303$ as in Casares et al. (2005). The observations of Table 1 and Table 2 are shown along the orbit. Figure 5 (a) and Fig. 5 (b) show the radio and Xray observations, respectively. As shown in the lower panel of Fig. 6 ( 37 years of radio data, Massi \& Torricelli-Ciamponi 2016), the FWHM of radio outburst occurs in the orbital range $0.4-0.8$ with its peak value around $\sim 0.6$. Figure 5 (a) shows that this orbital range $(0.4-0.8)$ is well sampled. In fact, observation W1 is at phase 0.45 and observation P3 is at phase 0.81 . The good sampling between the orbital phases $0.4-0.8$, indicates that radio QPOs are always associated with radio outbursts, either during their onset or during their decay. Especially vital is the radio observation P3 (performed four days after the outburst peak, i.e., at the end of the radio decay 
phase) where the flux density remained quiescent at a flux level of $55 \mathrm{mJy}$ for a long interval of 10 hours and no QPOs were detected for the same. X-ray observations are more sparse. In Fig. 5 (b), two of the X-ray observations, $\mathrm{H} 1$ and $\mathrm{S} 3$, were taken close to the periastron. Both the observations are of non-detection of QPOs. These orbital phases are also rather displaced from those where the radio outburst occur. Observation S2 is at phase 0.67 , where statistically the radio outburst is on its decay. It is interesting that the X-ray observation $\mathrm{S} 2$ shows variability but no periodicity (i.e., no QPOs). The only X-ray QPO detections, $\mathrm{H} 2$ and S1, are at the orbital phases, 0.43 and 0.56 , respectively, i.e., well in the range where statistically the onset of the radio outburst occurs.

The amplitude of the radio outburst of LS I $+61^{\circ} 303$ is modulated with the superorbital period. Top panel of Fig. 6 shows $\sim 37$ years of radio data folded with the long-term period $\left(P_{\text {long }}\right)$. The amplitude can change from the minimum flux density at $\Theta_{\min } \sim 0.6$ to the maximum flux density at $\Theta_{\max } \sim 0.1$ by a factor of 6 . Do we expect a dependence of QPO occurrence on the amplitude of the outburst? Figure 7 illustrates the dependence of superorbital phase and orbital phase for all the reported observations of Table 1 and Table 2. Detections seem to be independent of $\Theta_{\min }$ or $\Theta_{\max }$, i.e., they are independent from the amplitude of the radio outbursts. This result would be consistent with the precessing jet scenario of LS I $+61^{\circ} 303$ (Massi \& Torricelli-Ciamponi 2014). In this scenario, the superorbital modulation is not related to an intrinsic flux density enhancement but only depends on the Doppler boosting effects (i.e., apparent flux density enhancement).

\section{CONCLUSIONS AND DISCUSSION}

We presented here radio data of LS I $+61^{\circ} 303$ observed with the WSRT for four consecutive days in 2011. We also used the archival X-ray data of LS I $+61^{\circ} 303$ observed with the Suzaku satellite in 2009 for three different epochs. The following are our conclusions:

1. Our timing analysis establishes the presence of significant radio QPOs of $\sim 55$ minutes stable for the entire observation session of four days. This is the first time that short-time-scale radio QPOs are observed for such a long duration of time, in fact the $\sim 1.4$ hours microflares observed by Peracaula et al. (1997) were observed for $\sim 8$ hours. After the hints of X-ray variability in LS $\mathrm{I}+61^{\circ} 303$ by Harrison et al. (2000), we confirm here for the first time X-ray QPOs of $\sim 2$ hours period over a duration of 21.3 hours.

2. From the comparison between our work and previous QPO observations of $\mathrm{LS} \mathrm{I}+61^{\circ} 303$, there seems to be no dependence of the presence of QPOs on superorbital phase. Even though the good sampling of the orbital interval $0.4-0.8$ allows us to localise the presence of radio QPOs to both the onset and the decay phase of the radio outbursts. The more sparse X-ray observations seem to indicate the presence of X-ray QPOs only during the onset of the radio outburst.

3. We further note that QPOs in LS I $+61^{\circ} 303$ show different periods (from $\sim 1000$ seconds to $\sim 15$ hours) at different epochs.

In GRS 1915+105, QPOs have been interpreted as ejection of relativistic plasma clouds or plasmoids (Mirabel \& Rodríguez 1999, see their Fig. 7). Recently, magnetic reconnection events are invoked to explain the ejection of plasmoids in the jets of blazars, wherein current sheet is fragmented into a chain of plasmoids. These plasmoids can be of different size and can be ejected with different time-scales (minutes, hours to days) (e.g. Petropoulou et al. 2016; Sironi et al. 2016). Such magnetic reconnection events can thus give rise to QPOs of different periods in the radio jet and can significantly modulate the main outburst. Moreover, magnetic reconnection can also occur in the highly magnetised X-ray emitting accretion flow producing plasmoids and giving rise to QPOs at X-ray wavelengths. These plasmoids further expand adiabatically after leaving the accretion flow and could be seen as optically thin emission in the radio band (Yuan et al. 2009). In this second scenario, i.e., plasmoids generated within the accretion flow, magnetic reconnection could be the physical process creating the delayed radio QPOs with respect to the X-ray QPOs of same timescales, as observed in GRS 1915+105 (e.g. Mirabel \& Rodríguez 1999; Klein-Wolt et al. 2002). Future observations of LS I +61³03 should establish and provide a better understanding if the X-ray QPOs are present only during the onset of the radio outburst. Does this imply that X-ray QPOs are related solely to the ejection phase of plasmoids from the accretion flow? Are there associated delayed radio QPOs? Are the radio QPOs associated to the decay of the outburst still related to X-ray QPOs or are they just the result of magnetic reconnection occurring directly in the jet, as discussed above for blazars? These issues should be addressed by future observations.

\section{ACKNOWLEDGEMENTS}

We thank Frederic Jaron and Sergio A. Dzib for carefully reading the manuscript. The Westerbork Synthesis Radio Telescope is operated by the ASTRON (Netherlands Institute for Radio Astronomy) with support from the Netherlands Foundation for Scientific Research (NWO). This research has made use of data obtained from the Suzaku satellite, a collaborative mission between the space agencies of Japan (JAXA) and the USA (NASA). This research has made use of data and/or software provided by the High Energy Astrophysics Science Archive Research Center (HEASARC), which is a service of the Astrophysics Science Division at NASA/GSFC and the High Energy Astrophysics Division of the Smithsonian Astrophysical Observatory.

\section{REFERENCES}

Belloni T., Méndez M., King A. R., van der Klis M., van Paradijs J., 1997, ApJ, 479, L145

Casares J., Ribas I., Paredes J. M., Martí J., Allende Prieto C., 2005, MNRAS, 360, 1105

Chernyakova M., Babyk I., Malyshev D., Vovk I., Tsygankov S., Takahashi H., Fukazawa Y., 2017, MNRAS, 470, 1718

Fender R. P., Pooley G. G., Robinson C. R., Harmon B. A., Zhang S. N., Canosa C., 1997, in Wickramasinghe D. T., Bicknell G. V., Ferrario L., eds, Astronomical Society of the Pacific Conference Series Vol. 121, IAU Colloq. 163: Accretion Phenomena and Related Outflows. p. 701 (arXiv:astro-ph/9612092)

Fender R. P., Rayner D., Trushkin S. A., O'Brien K., Sault R. J., Pooley G. G., Norris R. P., 2002, MNRAS, 330, 212

Frail D. A., Hjellming R. M., 1991, AJ, 101, 2126

Gregory P. C., 2002, ApJ, 575, 427

Han X., Hjellming R. M., 1992, ApJ, 400, 304

Harrison F. A., Ray P. S., Leahy D. A., Waltman E. B., Pooley G. G., 2000, ApJ, 528, 454

Jaron F., Massi M., 2013, A\&A, 559, A129

Jaron F., Sharma R., Massi M., Fuhrmann L., Angelakis E., Myserlis I., Li G.-X., Shi X., 2017, MNRAS, 471, L110

Klein-Wolt M., Fender R. P., Pooley G. G., Belloni T., Migliari S., Morgan E. H., van der Klis M., 2002, MNRAS, 331, 745 
Linnell Nemec A. F., Nemec J. M., 1985, AJ, 90, 2317

Lomb N. R., 1976, Ap\&SS, 39, 447

Marti J., Mirabel I. F., Rodriguez L. F., 2001, Information Bulletin on Variable Stars, 5127

Massi M., Torricelli-Ciamponi G., 2014, A\&A, 564, A23

Massi M., Torricelli-Ciamponi G., 2016, A\&A, 585, A123

Massi M., Migliari S., Chernyakova M., 2017, MNRAS, 468, 3689

Mirabel I. F., Rodríguez L. F., 1999, ARA\&A, 37, 409

Mirabel I. F., Dhawan V., Chaty S., Rodriguez L. F., Marti J., Robinson C. R., Swank J., Geballe T., 1998, A\&A, 330, L9

Peracaula M., Marti J., Paredes J. M., 1997, A\&A, 328, 283

Petropoulou M., Giannios D., Sironi L., 2016, MNRAS, 462, 3325

Pooley G. G., Fender R. P., 1997, MNRAS, 292, 925

Ray P. S., Foster R. S., Waltman E. B., Tavani M., Ghigo F. D., 1997, ApJ, 491, 381

Rodríguez L. F., Mirabel I. F., 1997, ApJ, 474, L123

Scargle J. D., 1982, ApJ, 263, 835

Sironi L., Giannios D., Petropoulou M., 2016, MNRAS, 462, 48

Stellingwerf R. F., 1978, ApJ, 224, 953

Taylor A. R., Kenny H. T., Spencer R. E., Tzioumis A., 1992, ApJ, 395, 268

Yang Q.-X., Xie F.-G., Yuan F., Zdziarski A. A., Gierlinski M., Ho L. C., Yu Z., 2015a, Publication of Korean Astronomical Society, 30, 565

Yang Q.-X., Xie F.-G., Yuan F., Zdziarski A. A., Gierliński M., Ho L. C., Yu Z., 2015b, MNRAS, 447, 1692

Yuan F., Lin J., Wu K., Ho L. C., 2009, MNRAS, 395, 2183

Zimmermann L., Fuhrmann L., Massi M., 2015, A\&A, 580, L2

This paper has been typeset from a $\mathrm{T}_{\mathrm{E}} \mathrm{X} / \mathrm{L} \mathrm{T}_{\mathrm{E}} \mathrm{X}$ file prepared by the author. 